\title{
Parámetros del Múltiple de Escape para su Diseño Computacional: una revisión
}

\author{
Edwin R. Guamán ${ }^{(1,2)}$, Edilberto A. Llanes-Cedeño(1), Santiago F. Celi-Ortega ${ }^{(1,3)}$ y Juan C. Rocha-Hoyos ${ }^{(1) \star}$ \\ (1) Univ. Internacional SEK, Fac. de Arquitectura e Ingenierías, Programa Desarrollo Tecnológico UISEK, Casilla 17-03- \\ 02, Quito - Ecuador (e-mail: eguaman.mdm@uisek.edu.ec; antonio.llanes@uisek.edu.ec; santiago.celi@uisek.edu.ec; \\ carlos.rocha@uisek.edu.ec) \\ (2) Instituto Tecnológico Superior Central Técnico, Carrera de Mecánica Automotriz, Quito-Ecuador. \\ (3) Instituto Tecnológico Kachariy, Carrera de Mecánica Automotriz, Quito-Ecuador.
}

*Autor a quien debe ser dirigida la correspondencia.

Recibido Mar. 5, 2019; Aceptado Abr. 30, 2019; Versión final Jun. 28, 2019, Publicado Dic. 2019

\begin{abstract}
Resumen
El objetivo de este artículo fue analizar las variables de trabajo del múltiple de escape, a partir de una revisión de las distintas técnicas aplicadas a la modelación y validación, para determinarse los parámetros esenciales en la operación del múltiple de escape. El proceso definido se aplicó en función de métodos, variables de diseño, prestaciones y la tendencia actual en la dinámica de fluidos computacional. La utilidad del modelo se evaluó para la aplicación de optimización en función del componente objetivo, cálculos térmicos, condiciones de contorno, materiales para su análisis estructural y a fatiga, de forma integral. Por tanto, un diseño tubular posee mejores características de evacuación de los gases comparado con los colectores convencionales. El trabajo representa un aporte integral para mejorar el desempeño del vehículo. Además, se generó una propuesta para el diseño rápido y eficiente del múltiple escape.
\end{abstract}

Palabras clave: método de simulación; dinámica de fluidos computacionales; múltiples de escape; parámetros del modelo; análisis estructural

\section{Parameters of an Exhaust Manifold for its Computational Design. A Review}

\begin{abstract}
The objective of this article was to analyze the working variables of an exhaust manifold, from a review of the different techniques applied to modeling and validation, to determine the essential parameters in the operation of the exhaust manifold. The defined process was applied based on methods, design variables, performance and the current trend in computational fluid dynamics. The utility of the model was evaluated for the application of optimization based on the objective component, thermal calculations, boundary conditions, materials for structural analysis and fatigue, in an integral manner. Therefore, a tubular design has better characteristics of gas evacuation compared to conventional collectors. This works represents a contribution for improving the performance of the vehicle. Additionally, a proposal for the rapid and efficient design of the multiple exhaust was generated.
\end{abstract}

Keywords: simulation method; computational fluid dynamics; exhaust manifolds; parameter model; structural analysis 


\section{INTRODUCCIÓN}

Desde la creación del motor de 4 tiempos por Nikolaus August Otto y Eugen Langen en 1876, se han incorporado muchas innovaciones como la incorporación de la electrónica para los elementos de control del motor, mejoras en las propiedades de los aceites utilizados para la lubricación de elementos fijos y móviles, mejoras en la calidad y tipos de combustibles los cuales aportan con una explosión mucho más eficiente dentro de la cámara de combustión, lo que da como resultado una mejora general del rendimiento del motor (De Souza et al., 2018). En la mezcla de combustible y aire, la admisión de aire con la mayor eficiencia volumétrica se puede utilizar con la dosificación exacta, mediante un remolino adecuado el cual deberá poseer un número de Reynolds de 2000 a 3000 ya que un valor superior daría lugar a un flujo turbulento perjudicial para el ingreso de la mezcla (Rocha-Hoyos et al., 2018). La eficiencia volumétrica y una dosificación exacta permitirán obtener un mayor rendimiento del motor, economía en el consumo de combustible y disminución de las emisiones contaminantes. Los vehículos modernos se fabrican en su mayoría con motores turboalimentados, esto permite la inducción de la masa máxima de aire con la mayor eficiencia volumétrica posible y mediante el diseño de puerto apropiado de la entrada de aire, se logran los niveles de remolino requeridos para un mejor desempeño del motor. La mejora de las condiciones de flujo por prueba y error de un prototipo en el banco de flujo requiere de mucho tiempo y es muy costosa, incluso la fabricación de un colector de escape es una técnica bastante compleja que como todo proceso requiere de una normativa a seguir, la norma JIS G 4304, es la norma que gobierna la fabricación de este elemento del motor ya que esta maneja la manufactura de tiras de acero sin tensión según Park et al., (2006).

Las condiciones de flujo inestable dentro del cilindro dificultan la sintonización del flujo incluso utilizando técnicas sofisticadas (Latheesh et al., 2018; Van Basshuysen y Schäfer, 2018), por lo que la simulación 1D es una alternativa a las pruebas experimentales que permite obtener tiempos menores de simulación y ahorro de la capacidad de cálculo de un ordenador. La precisión que permite obtener al analizar velocidades de flujo o perfiles de distribución es una característica importante para utilizar esta técnica en el análisis de flujos para los elementos asociados al motor Desantes et al., (2011) así como el vaciado y llenado que se genera dentro de las cámaras y conductos asociados a la combustión Galindo et al., (2011). Los software OpenWAM y GTPower son herramientas que permiten realizar estos tipos de simulación.

Se han realizado muchas investigaciones para mejorar las condiciones de flujo dentro de la cámara de combustión utilizando un diseño diferente de los ductos de escape. Se observa que tanto los métodos numéricos como los experimentales están en buen acuerdo para la relación de turbulencia y el cálculo del coeficiente de flujo medio (Niyaz y Dhekane, 2014; Srinivasa et al., 2014). Por supuesto, el enfoque numérico debe validarse primero, lo que indica la necesidad de una buena base de datos experimental inicial (Decan et al., 2018; Rocha-Hoyos et al., 2017). La dinámica de fluidos computacional (CFD) es una herramienta que sirve para estudiar y respaldar la optimización del motor, esta permite un cambio rápido en la configuración del motor para determinar los diseños óptimos, sin tener que construir antes la configuración experimental. Específicamente, CFD es una técnica generalizada que surge del aumento significativo en la potencia de cálculo y la disponibilidad de códigos paralelos capaces de predecir el campo de flujo en geometrías complejas (Cai et al., 2015; Jeong y Seong, 2014).

Los gases de escape combustionados a temperaturas elevadas dentro de la cámara de combustión salen a altas velocidades hacia el colector por lo que el análisis estructural y térmico también son factores que se deben evaluar, así como las tensiones y desviaciones de la estructura del colector (Ekström y Jonsson, 2014). Los colectores de escape se diseñan para soportar temperaturas de hasta $1100{ }^{\circ} \mathrm{C}$ dependiendo el material del cuál estén fabricados, debido a que a las máximas potencias del motor, los gases de escape saldrán mucho más rápido de la cámara de combustión existiendo un menor tiempo de enfriamiento del colector (Gocmez y Deuster, 2009). Varios estudios se pueden encontrar en lo que se refiere al análisis de fluidos intervinientes en el funcionamiento del motor dentro de los cuales se usan software de simulación para calcular las variables como flujo, velocidad y presiones de entrada o de salida según sea la incógnita a despejar. Este artículo aborda las variables de trabajo del múltiple de escape, a partir de una revisión de los distintos modelos aplicados a la modelación y validación con el fin de la inclusión de los parámetros esenciales en la secuenciación de la optimización del múltiple de escape.

\section{TÈCNICAS DE SIMULACIÒN}

El presente estudio se realizó en varias etapas: la primera fase se centró en la definición del modelo matemático más común para la representación del múltiple de escape, empleando la propuesta metodológica de Valderrama, (2005). Como resultado se identificaron las ecuaciones que gobiernan el múltiple de escape y se establecieron las particularidades que rigen el colector. Al revisar la literatura encontrada se caracterizó el sistema de optimización a través de las técnicas predictivas 1D, de elementos finitos (FEM) y de análisis de dinámica en CFD dominantes en la ingeniería computarizada (CAE) (Vega et al., 2018). 


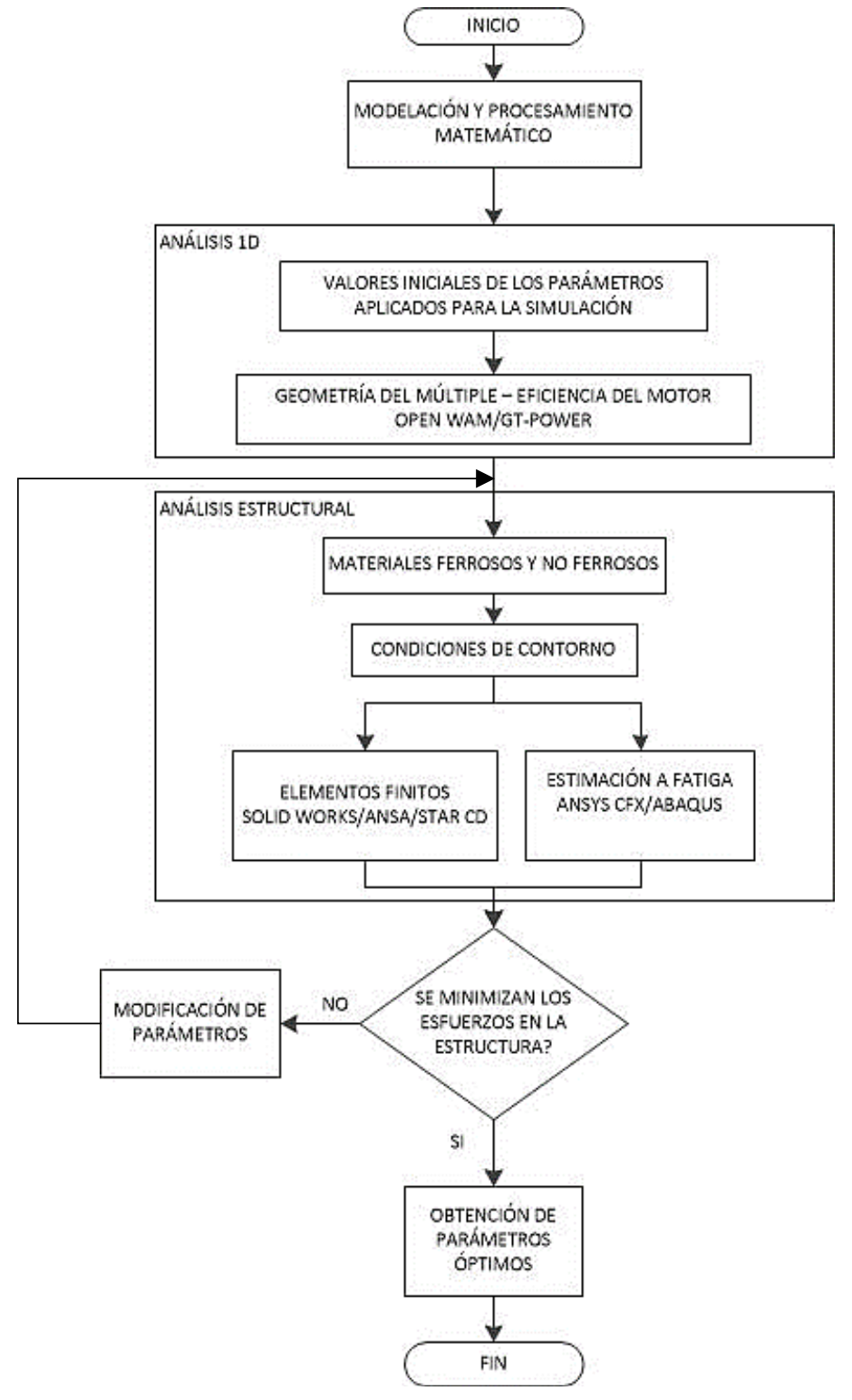

Fig. 1: Secuencia de investigación y optimización del múltiple de escape

En la figura 1, se muestra el proceso bibliográfico que se utilizó en el desarrollo del presente artículo. En el inicio se da una breve introducción y la metodología que se utilizó en la redacción, en la modelación y procesamiento matemático se conocieron las ecuaciones que rigen la geometría del colector y las magnitudes intervinientes en su funcionamiento, en el análisis 1D se determinaron los valores iniciales para la simulación y el análisis de la geometría para la selección de la mejor configuración, en el análisis estructural se determinaron los diferentes tipos de materiales y se distinguieron diferentes aleaciones de los cuales se fabrica esta autoparte, en las condiciones de contorno se mostraron los parámetros que utilizaron algunos autores para simular el desempeño y la durabilidad del diseño. Para finalizar, se identificaron las variables que permiten minimizar los esfuerzos sobre la estructura de colector y mejorar la eficiencia de circulación de los gases de escape para llegar a la obtención de los mejores parámetros para la optimización.

\section{LA MODELACIÓN Y PROCESAMIENTO MATEMÁTICO}

La contrapresión media está determinada principalmente por elementos simples, como el turbocompresor, el convertidor catalítico y el silenciador. La evolución instantánea de la presión impuesta por el sistema de escape en la válvula de escape, depende esencialmente del diseño y las dimensiones de las tuberías, por lo tanto, un diseño adecuado de la geometría del sistema de escape puede mejorar la potencia y la eficiencia del motor y reducir las emisiones de gases contaminantes. La contrapresión en el cilindro del motor depende completamente del diseño del sistema de escape, su condición de operación y la presión atmosférica a la que opera Patil et al., (2014). Existen múltiples diseños y condiciones de operación de los cuales se mencionan; múltiples de escape que usan chapas metálicas como blindaje térmico para proteger superficies y mandos de control del motor, son utilizados en motores para maquinaria pesada y tienen un costo elevado de adquisición, 
también están los múltiples de escape refrigerados por agua los cuales permiten la circulación de este líquido alrededor de la chapa interna de su estructura, logrando trabajar a temperaturas extremadamente bajas, induciendo que la chapa externa se expanda entre un 20 a un 40 por ciento, lo que no permite que un turbo compresor sea utilizable. Sabareesh et al., (2017) y Gocmez y Deuster, (2009) mencionan que también existen los múltiples de escape refrigerados por agua y aire ASWC (Air Shielded Water Cooled) en los cuales circula agua dentro del motor, que recolecta el calor del aire que envuelve la chapa interior del colector, lo que reduce la carga de enfriamiento del agua y mantiene una mayor vitalidad de los gases de escape que permiten que el turbocompresor sea más compatible (Rocha-Hoyos et al., 2019), pero posee un elevado costo y alta complejidad de construcción. Los colectores de tipo seco tienen un bajo costo, mejor trasmisión de calor, alcanza una temperatura superficial en menor tiempo de trabajo y son los que mejor vitalidad de gases de escape para el turbocompresor proporcionan (Sabareesh et al., 2017). Por lo que este es el tipo de múltiple de escape que se considera en esta revisión.

\section{Modelo numérico}

La morfología de un múltiple de escape consta de varios orificios de entrada o tubos de escape y un orificio de salida o colector de escape, debido al análisis que se requiere en este elemento se lo puede comparar con un miembro de una viga curva que sigue una trayectoria desde el origen $O$ hasta el centro de cada cilindro descritos como 1, 2, 3 y 4, por Park et al., (2006), en la cual cada cilindro comienza desde $\mathrm{X}_{\mathrm{R}}$, es decir, desde la izquierda de múltiple del colector, además se presenta una línea horizontal la cual describe una trayectoria rectilínea que recoge los tubos de escape de cada cilindro en un sistema de coordenadas $x, y, z$, que pasa por el centro de cada tubo de escape mostrada como un vector unitario $e_{u}=a_{i} e_{i}$. Por otra parte, la culata se toma como un punto de referencia para los desplazamientos y la ubicación de un punto extremo del colector $\mathrm{r}_{\mathrm{A}}{ }^{\mathrm{P}}$ como se muestra en la figura 2.

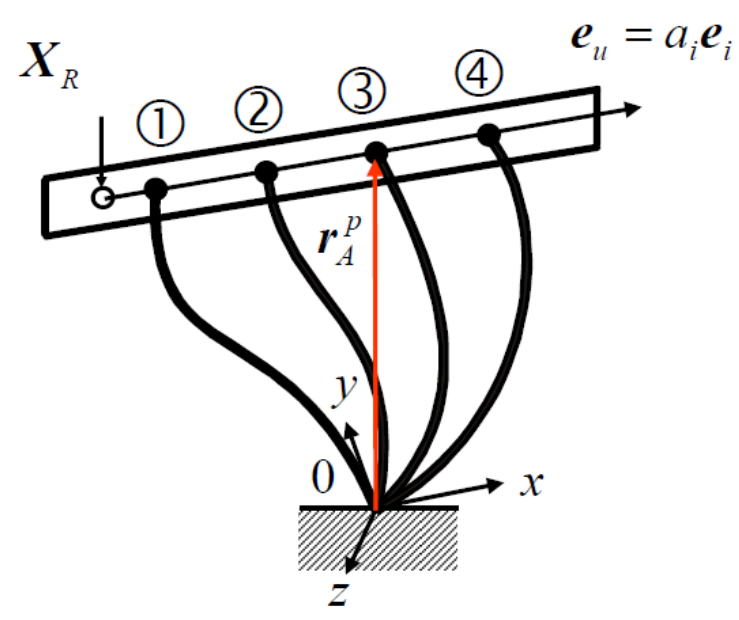

Fig. 2: Representación del múltiple de escape mediante vigas curvas (Adaptada de Park et al., 2006)

La ecuación que define la expansión térmica infinitesimal del múltiple de escape, se calcula de acuerdo a la ecuación 1 obtenida por Park et al.,(2006), donde du es la derivada del vector desplazamiento del tubo de escape, $\alpha$ es el coeficiente de expansión térmica del material constitutivo del tubo de escape, $T_{(s)}$ y $T_{r}$ son los coeficientes de expansión térmica del arco y desplazamiento del colector, $\mathrm{d}(\mathrm{s})$ es la derivada de la longitud desde el punto $\mathrm{O}$ al punto $B$ que se muestra en la figura 3 y dr(s) es la derivada de la posición del vector B.

$$
\left.d u=\alpha / T_{(s)}-T_{r}\right] d s \frac{d r(s)}{d s}
$$

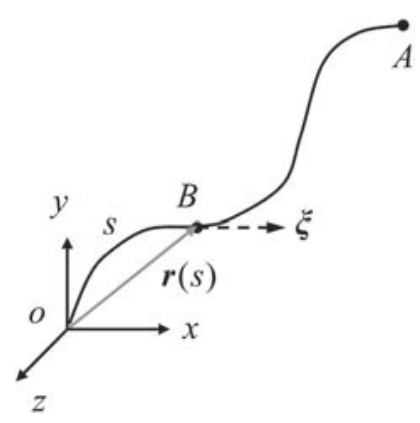

Fig. 3: Descomposición vectorial de cada tubo de escape con respecto a un sistema de coordenadas x, y, z. 
El colector de escape se puede aproximar a un miembro curvo con una longitud desde $O$ hasta $A$ según Park et al., (2006) considerando el punto $\mathrm{A}$ libre y el otro extremo fijo, donde $\mathrm{s}$ es la longitud desde el punto $\mathrm{O}$ hasta el punto $B, B$ es un punto arbitrario de la curva, $A$ es el punto de unión de la culata con el extremo de cada tubo de escape y $\xi$ es el vector unitario tangente a $\mathrm{B}$.

$$
U_{A}=\int_{0}^{A} d u=\alpha \int_{0}^{A} T_{(s)} \xi(s) d s-\alpha T_{R} \int_{0}^{A} \xi(s) d s
$$

A partir del supuesto que la distribución de las temperaturas es lineal se obtiene la ecuación 3 , donde $\mathrm{T}_{(\mathrm{s})}$ es la temperatura en el punto $B$, To es la temperatura en el origen $O, T_{A}$ es la temperatura en el punto $A$, s es la longitud desde el punto $\mathrm{O}$ al punto $\mathrm{B}$ y $\mathrm{I}$ es la longitud total entre los puntos de unión.

$$
T_{(s)}=T_{O}+\left(T_{A}-T_{O}\right) \frac{s}{l}
$$

Por último para obtener la geometría del colector $g_{A}$ se plantea la ecuación diferencial que representa el desplazamiento desde el punto $O$ hasta el punto $A$, de $r(s)$, donde $r(s)$ es una función polinomial de $s$, como se muestra en la ecuación 4 planteada por Park et al., (2006).

$$
g_{A}=\frac{1}{2} \int_{0}^{A} r(s) d s
$$

Por otra parte, también se analizan los diferentes factores que afectan al múltiple de escape desde diferentes partes de su estructura, dentro de las cuales se tiene la superficie interna, externa y de contacto, las que se detallan a continuación: Superficie interna.- Lederer et al., (2000) afirma que el calentamiento del múltiple de escape se genera por la convección forzada de los gases de escape y que la estimación de los coeficientes de convección local necesitan del conocimiento exacto de flujo del gas de escape sobre las paredes de la estructura, de igual manera Chinouilh et al., (2007) menciona que en el interior del múltiple de escape generalmente aparecen grietas en los extremos del domo o curvatura del tubo de escape las cuales reducen la vida útil del mismo.

Superficie externa.- Para la superficie externa se toma el coeficiente de convección que relaciona tanto la pared del colector con la temperatura del aire circulante, lo cual genera la transmisión de calor hacia el exterior, por esta razón Chinouilh et al., (2007) menciona que en el exterior del múltiple de escape se generan grietas múltiples con oxidación producida por el calor y que también generan vacíos debido a la cavitación.

Superficies de contacto.- El material que conforma el múltiple de escape es un componente sólido que genera transferencia de calor por lo que en las superficies de contacto, la conductancia del múltiple de escape con la culata se debe tomar muy en cuenta por su proximidad. Además, se debe de tomar en cuenta las condiciones de contorno a las cuales se somete durante su funcionamiento (Lederer et al., 2000).

\section{ANÀLISIS 1D}

Los modelos 1D permiten tratar los efectos inerciales en las secciones transversales de los orificios de entrada y salida de forma independiente, también permiten introducir los valores de los volúmenes de entrada y salida. Además, ofrecen más información sobre la velocidad de flujo o los perfiles de distribución de hollín, la regeneración, el análisis de gradiente de temperatura, etc., que muestran una alta precisión y fiabilidad (Desantes et al., 2011). Un sistema de admisión o escape se compone principalmente de conductos que pueden simularse con precisión mediante códigos unidimensionales no viscosos y son una opción correcta para ahorrar tiempo de cómputo, simulando componentes complejos mediante un código 3D y simulando los conductos con un código 1D. OpenWAM es un modelo dinámico de gas unidimensional de código abierto capaz de calcular los flujos de aire y gas dentro de los sistemas de admisión y escape de los motores de combustión interna. También es posible calcular el proceso de llenado y vaciado en cilindros, cámaras, turbinas, intercambiadores de calor y otros elementos por medio de un modelo concentrado. El cálculo de los elementos donde el flujo no es unidimensional se lleva a cabo utilizando ecuaciones de conservación simplificadas sin resolución espacial, que por lo general son casi constantes, además OpenWAM es particularmente adecuado para el cálculo del movimiento de la onda de presión en las tuberías de admisión y escape que afectan el rendimiento del motor. Sin embargo, las simplificaciones de las condiciones de contorno pueden llevar a resultados de baja precisión (Galindo et al., 2011). GT-Power también es un software de simulación de motores, utilizada por los principales fabricantes de motores y vehículos la cual proporciona muchos componentes a modelar para cualquier concepto avanzado, su facilidad de uso es una cualidad por la cual es muy utilizado (Vaidya y Hujare, 2014).

Arroyo et al., (2017) utilizó el software OpenWAM para el análisis de dos tipos de configuración de múltiples de escape, manteniendo constante el sistema que representa el múltiple de admisión y cilindros del motor. En la 
figura 6, se representa la primera configuración adoptada por este autor, en la que el colector de configuración 41 ayuda a disminuir la turbulencia, reducir pérdidas de flujo y teóricamente tiene el mayor caudal, por lo que trabaja mejor en altas revoluciones. Además, permite incrementar la velocidad de salida de los gases combustionados con lo que se logra obtener mayores valores de potencia a altas revoluciones y un aumento del torque con respecto al sistema original del $8.83 \%$.

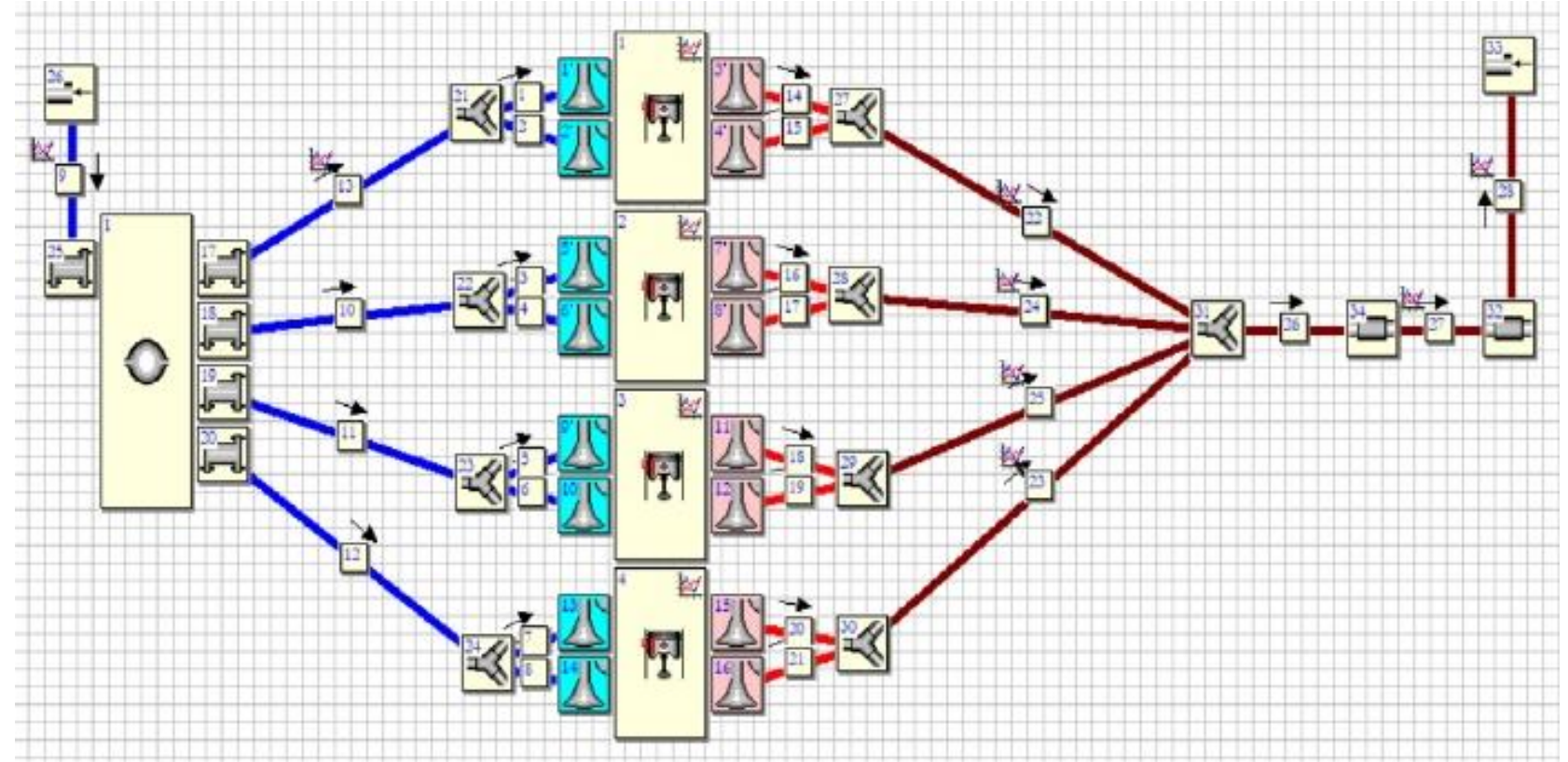

Fig. 6: Configuración 4-1 en OpenWAM (Adaptada de Arroyo et al., 2017).

\section{ANÁLISIS ESTRUCTURAL}

Los colectores están sometidos a temperaturas extremas. Las temperaturas pueden ir desde los $20{ }^{\circ} \mathrm{C}$ hasta llegar a los $950{ }^{\circ} \mathrm{C}$ y se pueden enfriarse nuevamente hasta la temperatura de $20^{\circ} \mathrm{C}$ que es la temperatura ambiente que rodea al colector, tales fases de calentamiento y enfriamiento crearán elevadas tensiones y deformaciones en su estructura. Las tensiones, esfuerzos y deformaciones máximas dependerán en gran medida del material, el diseño, la temperatura y la no linealidad de la geometría (Lakshmikantha y Kec, 2002).

\section{Materiales}

Los materiales de los que se fabrica un múltiple de escape son muy importantes debido a las altas temperaturas que se generan dentro del mismo. Babali y Seetharamu (2017) mencionan que los colectores de escape están hechos de cualquier forma de acero o de hierro fundido y que la mayoría usa hierro fundido debido a sus costos relativamente bajos y larga duración, Delprete y Rosso (2005) utilizaron colectores de escape fabricados de hierro fundido NiSiCr 35-5-2 y hierro fundido SiMoCr en dos casos de simulación, de los cuales el hierro fundido SiMoCr es el que resiste mejor a la fatiga térmica. Zeytin et al., (2009) afirma que los hierros dúctiles exhiben menos crecimiento a altas temperaturas debido a la estabilidad de la microestructura la cual es una aleación de silicio y molibdeno de grado ferrítico, que mejora el rendimiento del múltiple de escape. Lederer et al., (2000) en cambio utilizó un material plástico-elasto-viscoso el cual es resistente a temperaturas de 20 a $700{ }^{\circ} \mathrm{C}$ por lo que no es muy aplicable debido a que un colector podría superar estas temperaturas.

Por otro lado Gocmez y Deuster (2009) utilizan 16 tipos de materiales, de los cuales 8 son moldeados con materiales tales como: GJS-SiMo-5.0-1.0, GJS-SiMo-4.5-0.6, GJSA-XNiSiCr35-5-2, GX40CrNiSi25-20, los que resisten temperaturas de $700^{\circ} \mathrm{C}-830^{\circ} \mathrm{C}, 800^{\circ} \mathrm{C}-860^{\circ} \mathrm{C}, 850^{\circ} \mathrm{C}-1050^{\circ} \mathrm{C}, 1050^{\circ} \mathrm{C}-1100^{\circ} \mathrm{C}$ respectivamente con las ventajas de menor peso y menor cantidad de emisiones contaminantes pero con las desventajas de mayores costos y menor flexibilidad de diseño. También utilizó 8 materiales manufacturados industrialmente como el X2CrTiNb18, X5 CrNi 18-10, X6 CrNiTi 18-10, X15CrNiSi 20-12, que resisten temperaturas de $900{ }^{\circ} \mathrm{C}-950^{\circ} \mathrm{C}$, $800^{\circ} \mathrm{C}-850^{\circ} \mathrm{C}, 850^{\circ} \mathrm{C}-920^{\circ} \mathrm{C}, 950^{\circ} \mathrm{C}-1000^{\circ} \mathrm{C}$ respectivamente con la ventaja de una menor cantidad de emisiones contaminantes, más estabilidad térmica, menos carga dinámica y un diseño menos complejo. En la figura 4 se muestra una evaluación comparativa a partir de una base de datos, dentro de las cuales se usan compuestos de silicio y molibdeno con hierro dúctil, lo que permite ampliar la vida del material al hacerlos más resistente a fatiga térmica y reducir los efectos de la fluencia. 


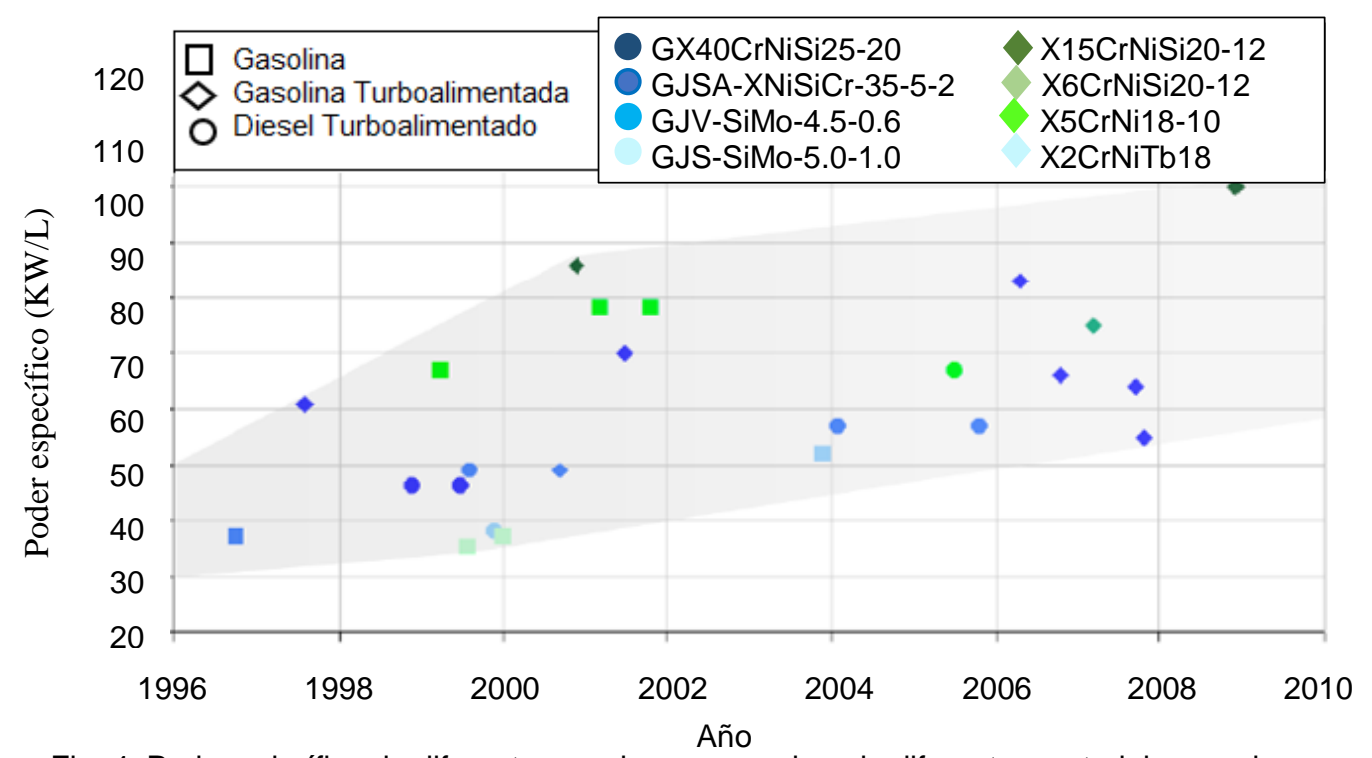

Fig. 4: Poder calorífico de diferentes combusuvies unizando diferentes materiales en el colector de escape (Adaptada de Gocmez y Deuster, 2009).

Por último (Santacreu et al., 2006; Chinouilh et al., 2007) afirman que el hierro fundido del que se fabricaban los múltiples de escape ha sido sustituido por el acero inoxidable, debido a que tiene una baja densidad y puede tolerar altos grados de fatiga térmica. Los aceros más utilizados son el acero ferrítico AISI 409 que soporta hasta $850^{\circ} \mathrm{C}$ y el acero ferrítico AISI 441 el cual soporta hasta $950^{\circ} \mathrm{C}$, mientras que los aceros inoxidables austeníticos, AISI 304 o AISI 321 son muy poco usados debido a que tienen algunas desventajas como la debilidad de resistencia a la oxidación cíclica y un alto coeficiente de expansión térmica; adicional a estos aceros también se usa el acero de grado austenítico refractario AISI 308, el cual es usado para trabajar a altas temperaturas.

\section{Condiciones de contorno}

Con respecto a las condiciones que diferentes autores establecieron para realizar el modelado y simulación se destaca el trabajo de Umesh et al., (2013) que evaluó en 8 ocasiones los gases de combustión, manteniendo una velocidad constante del motor a $1500 \mathrm{rpm}$, estos se tomaron a diferente carga; $2 \mathrm{~kg}, 4 \mathrm{~kg}, 6 \mathrm{~kg}, 8 \mathrm{~kg}, 10 \mathrm{~kg}, 12$ $\mathrm{kg}$ respectivamente, utilizando una sola cantidad de flujo másico en la entrada de los tubos de escape, con lo que obtuvo diferentes presiones y velocidades de flujo en la salida. El modelo lo realizó en Solid Works y el modelo numérico lo realizó en el software Ansys 12.0. Zeytin et al., (2009) en cambio utiliza condiciones de contorno con valores de flujo másico constante y varía las temperaturas y presiones a la salida del colector, además establece el material del colector como una pared no deslizante con un coeficiente de transferencia de calor de $45 \mathrm{~W} / \mathrm{m}^{2}$ con una rugosidad de $0.00508 \mathrm{~mm}$. Utilizó una malla tetraédrica refinada y aplicó el modelo de turbulencia Képsilon para las paredes cercanas del colector. De igual manera Hassan et al., (2014) uso diferentes flujos de entrada que fueron probados para dos configuraciones de múltiples de escape diferentes, estos valores fueron de 500,750 y $1000 \mathrm{~L} / \mathrm{min}$. Considerando la temperatura del agua a $20^{\circ} \mathrm{C}$ y una presión de salida de cero, pero variando el número de Reynolds de $10 \times 10^{4}, 15 \times 10^{4}$ y $20 \times 10^{4}$ respectivamente.

Por otro lado Sweta y Alkabani (2013) consideran que los gases de combustión calientes que fluyen en el interior del colector de escape tienen una temperatura máxima de $670{ }^{\circ} \mathrm{C}$ y asumen un coeficiente de transferencia de calor en el ingreso de $70 \mathrm{~W} / \mathrm{m}^{2}{ }^{\circ} \mathrm{C}$ y consideran una temperatura ambiente a la salida del colector de $25^{\circ} \mathrm{C}$, $35^{\circ} \mathrm{C}$ y $50^{\circ} \mathrm{C}$ que cubren la mayoría de temperaturas posibles hasta la máxima que se podría alcanzar en el final del colector, en este punto también se considera un coeficiente de transferencia de calor exterior de $30 \mathrm{~W} / \mathrm{m}^{2}{ }^{\circ} \mathrm{C}$, el análisis se realiza para el mapeo térmico sobre el cuerpo completo que permitirá calcular todos los valores térmicos nodales los cuales dependen de la resistencia térmica de los materiales, este mapeo de temperatura en el colector es la transferencia térmica que se suscita en la estructura del mismo. Un año después Navadagi y Sangamad (2014) utilizan 2 modelos de tubos de escape, uno estándar o inicial y el otro modificado con un diámetro de $42 \mathrm{~mm}$ y una longitud de $0.6 \mathrm{~m}$ para realizar la simulación a 4 diferentes velocidades del motor 1300 , 1700,1800 y $1900 \mathrm{rpm}$ a diferentes flujos $3.143,4.516,4.981$ y $5.627 \mathrm{~m}^{3} / \mathrm{s}$ respectivamente, obteniendo que la contrapresión es menor en el múltiple modificado que en el inicial, según muestra la figura 5. Selvanathan et al., (2017) también utilizó aire en tres modelos de colectores con turbo cargador, manejando una entrada de flujo másico de $0.0482 \mathrm{~kg} / \mathrm{s}$, una presión de salida de $0 \mathrm{~Pa}$., todo esto a $60000 \mathrm{rpm}$ y lo simuló en el software ANSA 13.2, obteniendo que, un diseño tubular mejora la velocidad de salida del aire en hasta $254 \mathrm{~m} / \mathrm{s}$ comparados con los $210 \mathrm{~m} / \mathrm{s}$ y $191 \mathrm{~m} / \mathrm{s}$ obtenidos con colectores convencionales, lo que comprueba que un diseño eficiente del colector de escape juega un papel muy importante en la mejora de la eficiencia del motor. 


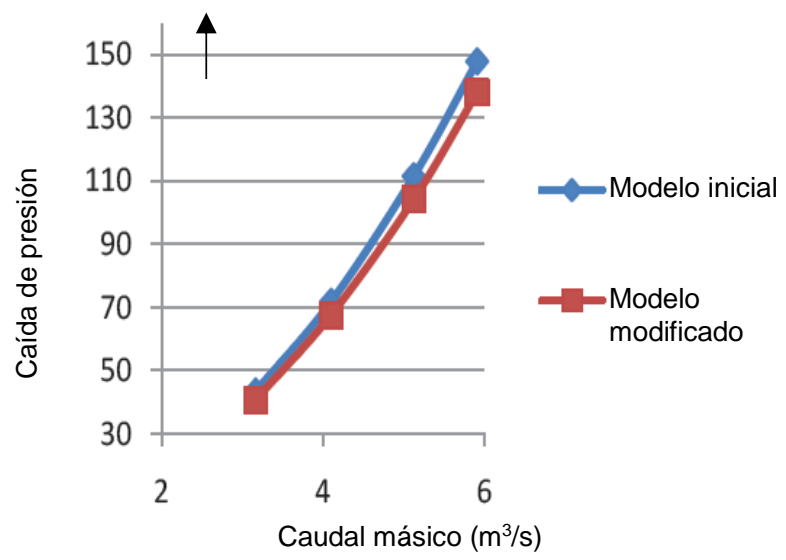

Fig. 5: Comparación de contrapresión en un modelo estándar y modificado (Adaptada de Navadagi y Sangamad, 2014)

Yoshizawa et al., (2001) utilizó un fluido comprensible asumiendo el flujo de gases de escape y el coeficiente de transferencia de calor, para simular el comportamiento térmico del colector de escape y del panal del catalizador, con lo que obtienen que la simulación unidimensional y tridimensional concuerdan con los valores obtenidos en los métodos experimentales, así mismo, Babali y Seetharamu (2017) utilizaron un fluido con una densidad de $1.045 \mathrm{~kg} / \mathrm{m}^{3}$, viscosidad de $3.0927 \times 10^{5} \mathrm{~Pa}-\mathrm{s}$, calor específico de $1056.64 \mathrm{~J} / \mathrm{kg}-\mathrm{K}$ y una conductividad térmica de $0.0250 \mathrm{~W} / \mathrm{m}-\mathrm{K}$ para tres velocidades diferentes de 100, 3000 y $6000 \mathrm{rpm}$ asumiendo un flujo másico en la entrada igual al flujo másico de la salida, una eficiencia volumétrica de $90 \%$, el flujo se considera turbulento lo que demostró que un modelo diseñado con una geometría diferente produce la menor contrapresión. Por otra parte, Umesh et al., (2013) utilizó velocidades de flujo másico de $0.001696 \mathrm{~kg} / \mathrm{s}$ para $2 \mathrm{~kg}, 0.003392 \mathrm{~kg} / \mathrm{s}$ para $4 \mathrm{~kg}$, $0.005088 \mathrm{~kg} / \mathrm{s}$ para $6 \mathrm{~kg}, 0.006784 \mathrm{~kg} / \mathrm{s}$ para $8 \mathrm{~kg}, 0.008480 \mathrm{~kg} / \mathrm{s}$ para $10 \mathrm{~kg}$ y $0.10176 \mathrm{~kg} / \mathrm{s}$ para $12 \mathrm{~kg}$ todo esto para múltiples de escape de salida lateral de curva corta (SBSE) y para un modelo de salida central de curva larga (LBCE), utiliza en cambio en cambio un flujo másico de $0.0156 \mathrm{~kg} / \mathrm{s}$ para $1000 \mathrm{rpm}, 0.0469 \mathrm{~kg} / \mathrm{s}$ para 3000 rpm y $0.0939 \mathrm{~kg} / \mathrm{s}$ para $6000 \mathrm{rpm}$ en los modelos 1 (MM1), modelo 2 (MM2) y modelo 3 (MM3) adoptados, con lo que obtuvo que el modelo LBCE da un puntaje de rendimiento del $100 \%$ sobre otros modelos.

\section{Ecuaciones fundamentales}

En el análisis estructural se calculan las tensiones generadas por los campos térmicos y las cargas mecánicas tomando en cuenta la no linealidad del comportamiento del material, además de las condiciones de contorno reales que están representadas por restricciones y por contactos entre los componentes. Estas condiciones de restricción son las condiciones teóricas en las que el colector de escape puede funcionar en los extremos de su campo de trabajo. Las condiciones de restricción son útiles porque el modelo de FE (Elementos Finitos) simplificado es más fácil de obtener y más rápido de ejecutar con respecto al modelo de FE completo. El modelo FE completo representa la condición de trabajo más cercana a las condiciones de trabajo real del colector (Delprete y Rosso, 2005). En los gases de escape el flujo es tridimensional por lo que la velocidad del mismo se localiza en los tres ejes de coordenadas $x, y, z$. Las ecuaciones que rigen dicho movimiento se pueden representar con las ecuaciones diferenciales $5,6,7$ y 8 donde; 5 es la ecuación de conservación de masa y 6,7 y 8 son las ecuaciones de conservación de impulso, mientras que $\mathrm{u}, \mathrm{v}$ y $\mathrm{w}$ son las componentes dimensionales de la velocidad y p es la componente de la densidad de flujo Chen y Sparrow (2009).

$$
\frac{d u}{d x}+\frac{d v}{d y}+\frac{d w}{d z}=0
$$

Momentum en $X$

$$
\left.P / \frac{d}{d x}\left(u^{2}\right)+\frac{d}{d y}(u v)+\frac{d}{d z}(u w)\right]=-\frac{d p}{d x}+\frac{d}{d x}\left(\mu_{\text {eff }} \frac{d}{d x}\right)+\frac{d}{d x}\left(\mu_{\text {eff }} \frac{d u}{d y}\right)+\frac{d}{d z}\left(\mu_{\text {eff }} \frac{d u}{d z}\right)
$$

Momentum en $Y$

$$
P\left[\frac{d}{d x}(u v)+\frac{d}{d y}\left(v^{2}\right)+\frac{d}{d z}(u w)\right]=-\frac{d p}{d y}+\frac{d}{d x}\left(\mu_{\text {eff }} \frac{d v}{d x}\right)+\frac{d}{d y}\left(\mu_{\text {eff }} \frac{d v}{d y}\right)+\frac{d}{d z}\left(\mu_{\text {eff }} \frac{d v}{d z}\right)
$$

Momentum en Z

$$
\left.P / \frac{d}{d x}(w v)+\frac{d}{d y}(w v)+\frac{d}{d z}\left(w^{2}\right)\right]=-\frac{d p}{d z}+\frac{d}{d x}\left(\mu_{\text {eff }} \frac{d w}{d x}\right)+\frac{d}{d y}\left(\mu_{\text {eff }} \frac{d w}{d y}\right)+\frac{d}{d z}\left(\mu_{\text {eff }} \frac{d w}{d z}\right)
$$




\section{Calidad de malla}

La consideración más fundamental en CFD es cómo se trata un fluido continuo de manera discretizada en una computadora. Un método consiste en discretizar el dominio espacial en celdas pequeñas para formar una malla o cuadrícula de volumen, y luego aplicar un algoritmo adecuado para resolver las ecuaciones de movimiento. Además, dicha malla puede ser irregular (por ejemplo, consta de triángulos en 2D o sólidos piramidales en 3D); La característica de la primera malla, es que, cada celda debe almacenarse por separado en la memoria. La razón más importante para el incremento de la simulación CFD es que para muchas de las simulaciones de flujo convencionales, el CFD es significativamente más barato que las pruebas de túnel de viento y lo será aún más en el futuro. Las mejoras en hardware y procesamiento numérico de las computadoras han generado una plataforma para simular condiciones físicas extremas como un número de Reynolds más alto, una temperatura más alta, etc. Seshadri S., (2015). Mamiya et al., (2002) utilizó un tamaño de malla de $3 \mathrm{~mm}$ de aproximadamente 260000 celdas de malla y las condiciones de contorno las derivó del software GT-POWER para luego utilizarlas en el programa STAR-CD con lo cual realiza el cálculo del coeficiente de transferencia de calor de la pared interna del múltiple de escape. El análisis dinámico del gas, se realizó en un software desarrollado por Nissan, para calcular el coeficiente de transferencia de calor de la pared exterior. Una vez obtenidos estos datos se introdujeron como condiciones de contorno de un análisis de conducción térmica, para la derivación de la temperatura en condiciones de movimiento del vehículo. Lederer et al., (2000) en cambio realiza la simulación estructural, donde concluye que una malla de análisis de elementos finitos para el múltiple de escape tiene alrededor de 15000 elementos lineales correspondientes a 66000 grados de libertad, como se muestra en la figura 8 y que el acoplamiento entre la culata y el colector del escape se puede representar por un número de tornillos y un modelo de contacto deslizable pequeño.

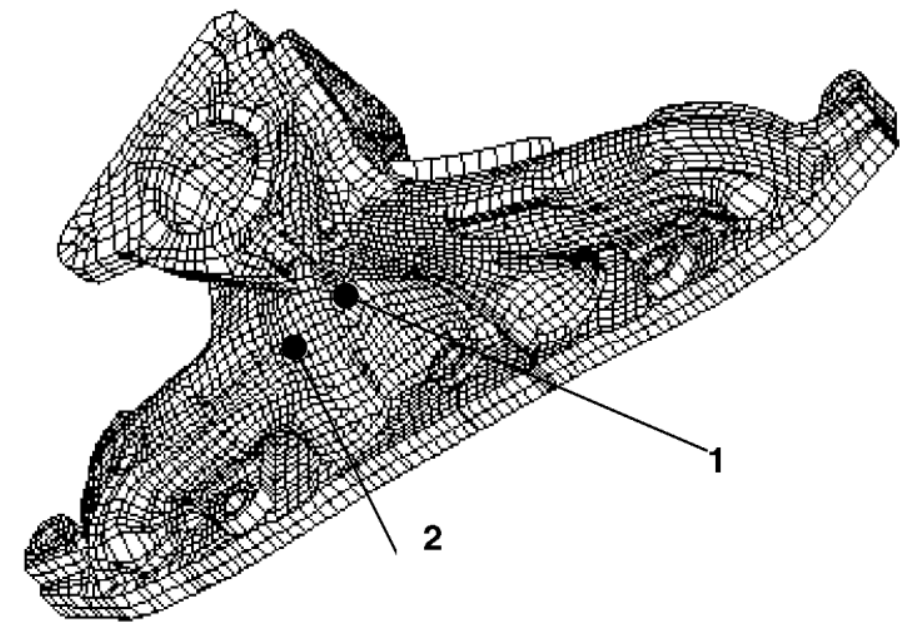

Fig. 8: Modelado del múltiple de escape (Adaptada de Lederer et al., 2000)

Navadagi y Sangamad (2014) crearon una malla estructurada de bloques múltiples para realizar una simulación de flujo CFD bajo condiciones isotérmicas para gases de escape, para lo cual utilizaron el modelo de turbulencia k-épsilon teniendo en cuenta las altas velocidades y las fuertes curvaturas del flujo dentro del colector, en otros casos el mallado del colector fue de aproximadamente un millón de elementos por cada elemento geométrico. De estas experiencias podemos apreciar que, si se elige construir una malla computacional manualmente se puede realizar una malla hexaédrica, sin embargo, si la geometría es lo suficientemente complicada se necesita de un generador de malla automático y que para una simulación CFD una malla tetraédrica es una opción muy práctica Fan et al., (2004).

\section{Elementos finitos}

La estructura del múltiple de escape es la unión de varios tubos de escape con un colector, sin embargo, Park et al., (2006), relacionó las fuerzas (F) y los desplazamientos en un sistema elástico que tiene como resultado una matriz de desplazamiento generalizado para el tubo de escape $D$, donde la letra $C$ es una matriz $6 \times 6, F(i)$ es un componente de la fuerza $(1,2,3)$ la cual queda expresada de la siguiente manera: $D=C$.F. El esfuerzo en la cabeza del cilindro también es importante dentro de este estudio, por lo que se considera que las deformaciones de la culata debido a los momentos de flexión son despreciables, porque el módulo de flexión de la culata es mucho mayor que el de los tubos de escape, además se considera que la cabeza del cilindro solo se deforma en la dirección que se muestra en la figura 7, según Park et al., (2006), donde: k es la constate de elasticidad del resorte; $F$ es la fuerza en la unión de la cabeza del cilindro y el múltiple; u es el desplazamiento nodal del cabezote y del múltiple de escape. 


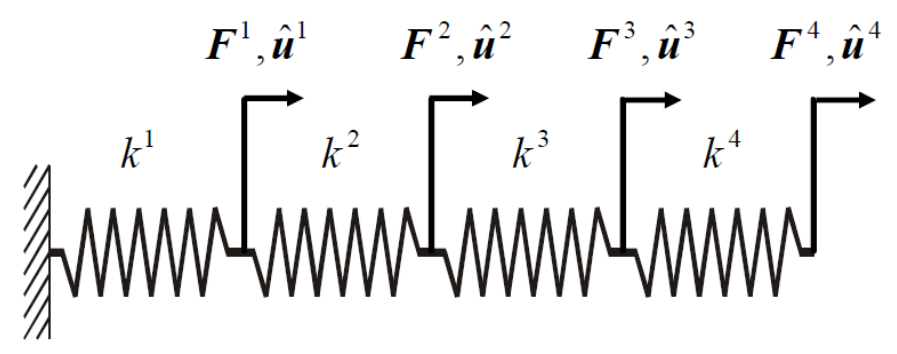

Fig. 7: Representación del múltiple de escape mediante resortes

Los nuevos modelos de motores son probados bajo una curva de presión y velocidad, la contrapresión generada dentro de los tubos de escape depende de la geometría de estos motores, una contrapresión mínima se tomará como la ideal y la que generará mayor eficiencia en el rendimiento del motor. Gopaal et al., (2014) incorpora los campos de la mecánica y la dinámica, así como algunas teorías de falla para este componente, las cargas que actúan sobre la estructura y las condiciones que soporta sirven para el análisis estructural del mismo debido a que en estos análisis se obtienen las reacciones en los soportes y los valores de tensión que se generan sobre este cuerpo. Gocmez y Deuster (2009) mencionan que la distribución de temperatura es la condición límite más importante para el análisis estructural y que durante el desarrollo de un motor se suelen usar diferentes niveles de valor límite térmico.

\section{Estimación a fatiga}

Gocmez y Deuster (2009) afirman que la condición más importante es la distribución de temperatura y que las condiciones de frontera provienen de diseños de similares características, que permitirán realizar una simulación 2D, la cual proporcionará condiciones de contorno más detalladas para la simulación 3D de CFD. Además de la temperatura se tienen que tomar en cuenta la radiación entre láminas interna y externa dentro de los colectores de escape de doble pared, así mismo Lederer et al., (2000) menciona que la mayor estimación de vida de los elementos mecánicos ha sido derivada del análisis de fatiga termo-mecánico al aplicar las pruebas térmicas sobre los múltiples de escape. De igual manera, Park et al., (2006) indica que el análisis para el estudio paramétrico debe incluir un análisis termo-elasto-plástico no lineal para evaluar la vida del elemento a fatiga y que se requiere una gran cantidad de tiempo en el procesamiento previo y posterior. El primer análisis térmico estudiado se realizó con el ajuste de las medidas experimentales mediante termopares, como se muestra en la figura 9 y se usó un proceso de uso del material necesario para mejorar la eficiencia del análisis termo-mecánico usando un elastovisco-plástico.

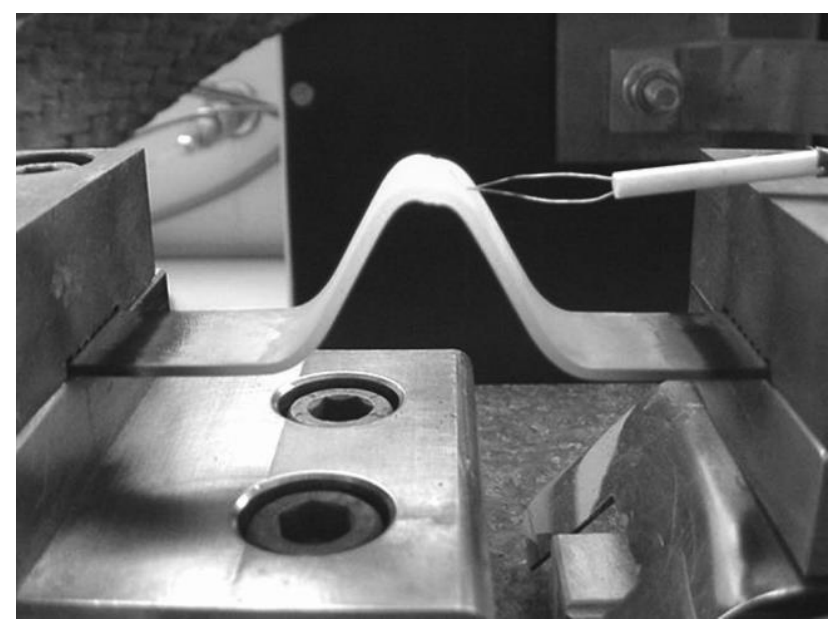

Fig. 9: Desarrollo de test a fatiga de un acero inoxidable (Adaptada de Santacreu et al., 2006)

Lederer et al., (2000) determinó que la predicción de tiempo de vida de los elementos mecánicos se debe analizar mediante la deviación estándar de los resultados experimentales considerada por el número de ciclos que la misma tiene que soportar, al respecto Gopaal et al., (2014) también menciona que se debe realizar un análisis modal sobre el colector para comprobar la respuesta de la estructura a cargas dinámicas en condiciones de resonancia. Finalmente, Navadagi y Sangamad (2014) indica que las altas presiones generadas en la entrada del múltiple de escape se deben a la diferencia de presión entre el múltiple y la cámara de combustión, mostrando dos modelos en los cuales el modelo original no distribuye uniformemente la presión generando un impacto negativo de la velocidad de flujo y el otro modelo mejorado genera un flujo de presión uniforme que genera un aumento de la velocidad de flujo. 
Park et al., (2006) menciona que es muy común en una etapa de diseño que la selección de material esté restringida y que la temperatura del gas en el colector de escape esté limitada a un cierto valor, debido a los factores que afectan el rendimiento de un motor, además que la manera útil de aumentar la durabilidad del colector de escape es un estudio paramétrico de la geometría con la temperatura y los materiales utilizados. Santacreu et al., (2006) utilizó el software ABAQUS como solucionador tanto para el análisis térmico como mecánico de las diferentes pruebas que realiza, como se observa en la figura 10, donde las temperaturas máximas, mínimas, el tiempo y el grado de fluencia fueron variados. Gopaal et al., (2014) realiza en cambio, dos tipos de análisis térmicos los cuales fueron el análisis térmico en estado estacionario y el análisis térmico transitorio donde se analizaron las condiciones térmicas estables en periodos no considerados y donde las condiciones térmicas variaban según el tiempo para las pruebas respectiva obteniendo como resultado que el múltiple de escape simulado soportaba satisfactoriamente las cargas asignadas.

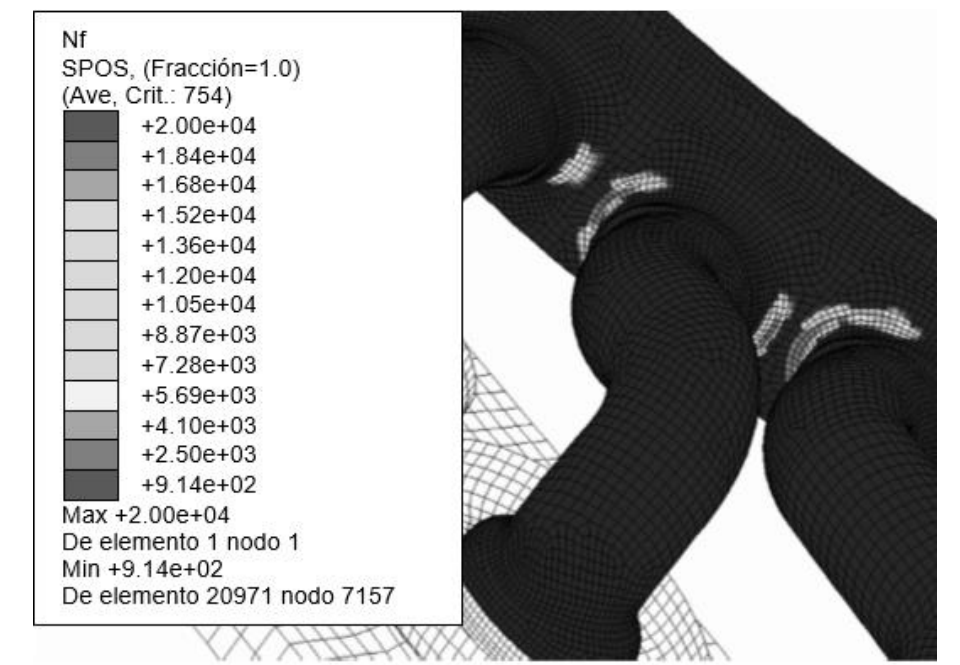

Fig. 10: Predicción del tiempo de utilidad del múltiple de escape a través del software ABAQUS (Adaptada de Santacreu et al., 2006)

Zeytin et al., (2009) aplicó una prueba de partículas magnéticas en el colector de escape para determinar problemas de porosidad, contracción, pequeñas y grandes grietas en el lado de la brida del turbo cargador y el colector fue cortado a lo largo y examinado metalográficamente para ver las grietas, las cuales se determinó que son paralelas y que se generan en la sección transversal completa. Durante la solidificación se formaron carburos en forma de aguja como se puede apreciar en la figura 11.

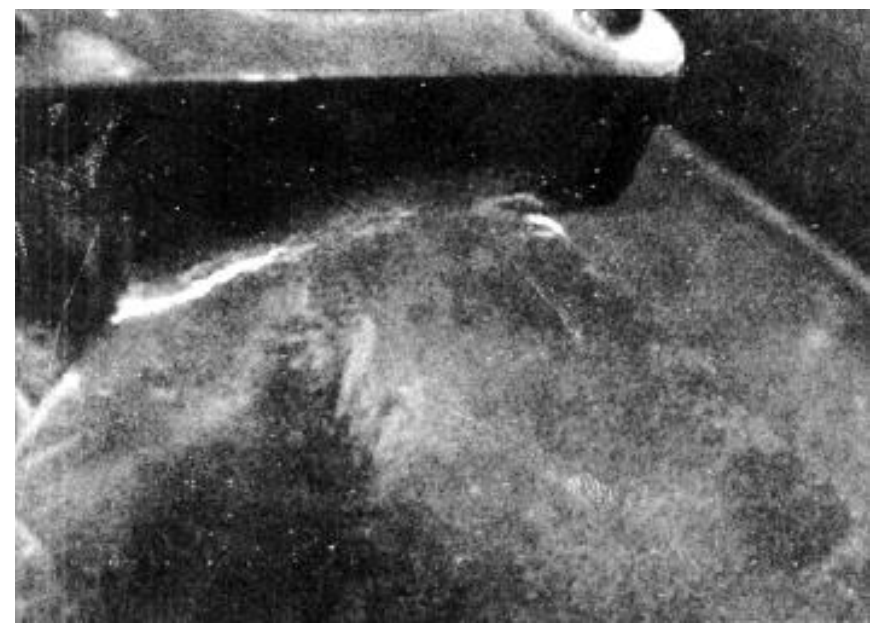

Fig. 11: Grietas generadas en el múltiple de escape

Chinouilh et al., (2007) afirma que la conversión catalítica aumenta la temperatura de los gases de escape y por ende aumenta la corrosión y la fatiga mecánica térmica por lo que tendría que usarse un acero inoxidable en lugar del hierro fundido. Además realizó una serie de pruebas en diferentes calidades de acero inoxidable, para temperaturas de $650^{\circ} \mathrm{C}$ a $1000{ }^{\circ} \mathrm{C}$ con 180 segundos de permanencia, donde mediante la metalografía se observa la iniciación de grietas en el interior y grietas múltiples más sectores en oxidación en la parte exterior, además de la formación de vacíos por la cavitación. Mamiya et al., (2002) realizó un análisis de conducción de calor transitorio para simular el funcionamiento y enfriamiento del ciclo de calor y también calculó el rango de 
deformación plástica del múltiple de escape en función de las distribuciones de temperatura usando el análisis térmico no lineal con lo cual se pudo predecir la vida a fatiga en el rango de deformación utilizando el software ABAQUS. Por último, Zeytin et al., (2009) enuncia que al aumentar la cantidad de molibdeno en la estructura del colector, aumenta la fatiga térmica y que el silicio aumenta la resistencia a la oxidación, además las condiciones de solidificación y enfriamiento son muy significativas porque determinan la cantidad de ferrita y perlita en los hierros, y que los tiempos de apertura y cierre del molde son parámetros muy importantes.

\section{RESULTADOS Y DISCUSION}

Babali y Seetharamu (2017) afirman que la selección del múltiple de escape es una decisión complicada y que se tienen que seleccionar tubos estrechos para una menor presión de retorno y que tuberías muy anchas darán lugar a contrapresión y habrá pérdida de potencia debido a un mal flujo de escape. Por tanto, si la velocidad del motor está dentro de las 2000 a 3000 rpm es una decisión adecuada las tuberías estrechas, sin embargo, cuando se encuentran alrededor de las 6000 rpm las tuberías anchas son las que generan un mejor rendimiento.

Gocmez y Deuster (2009) mencionan que en muchos casos el parámetro de influencia principal de cualquier característica de diseño del múltiple de escape tiene que ver con la fuerza del perno que sujeta el múltiple con el cabezote y que en el análisis inicial se tienen que incluir las pretensiones de perno mínimas y máximas adecuadas debido a que estas proporcionarán información relevante en la respuesta del sistema, y que además deben revisarse también los resultados con respecto a la presión de sellado de la junta y la vida útil de esta. Chen y Sparrow (2009) utilizan tres diferentes puertos de salida para su estudio, el uno de matriz de aberturas circulares discretas, otro con una matriz de ranuras rectangulares con filetes redondos y otro de ranura rectangular continua con lo que demostró que la geometría de la salida afecta a las tasas de flujo másico del colector de escape, siendo el colector de escape de ranura continua el que mejor rendimiento ofrece de extremo a extremo, aproximadamente de $+-5 \%$. Mientras que los colectores con ranuras rectangulares y circulares discretas proporcionan una uniformidad de +-10\% y +-15\% respectivamente. Mamiya et al., (2002) enuncia que se puede predecir la temperatura del múltiple de escape en condiciones de operación mediante un análisis cuantitativo, además Selvanathan et al., (2017) asegura que la longitud, el pulso de escape, la contrapresión y la forma física tienen un impacto significativo en la velocidad del escape y que el diseño tubular permite obtener una velocidad máxima de $254 \mathrm{~m} / \mathrm{s}$ de rendimiento adicional.

Por último, Arroyo (2017) indica que, los colectores con entradas rectas y radios de giro más amplios aumentan la velocidad de entrada al múltiple entre un $28 \%$ a $32 \%$ lo que permite una evacuación de gases con mayor rapidez, la configuración 4-1 además que es la más aconsejada debido a que aumenta la velocidad de salida de los gases de escape en un $16 \%$ con respecto al diseño original, mientras que el diseño 4-2-1 aumenta un $14 \%$ de esta velocidad con respecto al mismo diseño original del motor, además el incremento de potencia con un múltiple de configuración 4-1 con respecto al sistema original es de $8.41 \%$ a un $10.33 \%$ con múltiples modificados respecto al original y que además el par motor aumenta también de $3.26 \%$ a un $8.83 \%$ siendo un aporte muy significativo cuando las revoluciones del motor están sobre las $5000 \mathrm{rpm}$.

\section{CONCLUSIONES}

De la investigación realizada se concluye: 1) La aplicación de software de simulación para el análisis de los flujos en los múltiples de escape, es de gran importancia en la selección de la configuración y dimensionamiento del mismo. 2) Un diámetro de tubo de escape pequeño es aconsejado para motores que trabajarán en un rango de 2000 a 3000 rpm, mientras que para rpm mayores se aconseja un diámetro mayor. 3) Un diámetro de curvatura mayor de los tubos de escape permite mejorar la eficiencia de salida de los gases de escape. 4) En la construcción del múltiple de escape se han experimentado diferentes materiales a base de Cromo, Níquel, Silicio, Molibdeno, entre otros, llegándose a considerar los de acero e hierro fundido, prevaleciendo el uso del hierro fundido debido a sus costos relativamente bajos y larga duración. 5) El acero inoxidable AISI 409 y AISI 441 como material para múltiples de escape es el mejor candidato a reemplazar al comúnmente utilizado hierro fundido debido a su baja densidad y su alta resistencia a la fatiga térmica.6) Las ranuras circulares en la geometría del colector permiten un aumento de $\pm 15 \%$ de la velocidad del flujo. 7) A partir de los resultados obtenidos por diferentes autores en cuanto a las condiciones de contorno, se puede concluir que un diseño tubular posee mejores características de evacuación de los gases comparado con los colectores convencionales.

\section{REFERENCIAS}

Arroyo, E., Optimización del múltiple de escape para un vehículo de competición con motor de combustión interna de cuatro tiempos y cuatro cilindros (2017)

Babali, B., A. y K.N. Seetharamu, CFD Analysis of Exhaust Manifold of A multi-Cylinder Engine. International Journal of Electronics, Electrical and Computational System, 1-10 (2017) 
Cai, Y., Z. Liu, Q. Song, Z. Shi, y Y. Wan, Fluid mechanics of internal flow with friction and cutting strategies for micronozzles, doi: 10.1016/j.jjmecsci.2015.06.011, International Journal of Mechanical Sciences, 100, 41-49 (2015)

Chen, A. W., y E. M. Sparrow, Effect of exit-port geometry on the performance of a flow distribution manifold. Applied Thermal Engineering, 29(13), 2689-2692 (2009)

Chinouilh, G., P. O. Santacreu, y J. M. Herbelin, Thermal fatigue design of stainless steel exhaust manifolds (No. 200701-0564). SAE Technical Paper, 1-8 (2007)

De Souza, G. R., C. de Castro Pellegrini, S. L. Ferreira, F. S. Pau, y O. Armas, Study of Intake Manifolds of an Internal Combustion Engine: A New Geometry Based on Experimental Results and Numerical Simulations, doi:10.1016/j.tsep.2018.12.003, Thermal Sci. and Eng. Progress, (2018).

Decan, G., S. Broekaert, T. Lucchini, G. D’Errico, J. Vierendeels, y S. Verhelst, Evaluation of wall heat flux calculation methods for CFD simulations of an internal combustion engine under both motored and $\mathrm{HCCl}$ operation. Applied Energy, 232, 451-461 (2018)

Delprete, C., y C. Rosso, Exhaust manifold thermo-structural simulation methodology (No. 2005-01-1076). SAE Technical Paper (2005)

Desantes, J.M., J.R. Serrano, F.J. Arnau, y P. Piqueras, Derivation of the method of characteristics for the fluid dynamic solution of flow advection along porous wall channels, doi:10.1016/j.apm.2011.09.090, Applied Mathematical Modelling, 36(7), 3134-3152 (2012)

Ekström, M., y S. Jonsson, High-temperature mechanical-and fatigue properties of cast alloys intended for use in exhaust manifolds. Materials Science and Engineering: A, 616, 78-87 (2014)

Fan, Q.,Y., M. Kuba, y J. Nakanishi, Coupled Analysis of Thermal Flow and Thermal Stress of an Engine Exhaust Manifold, doi: 10.4271/2004-01-1345, SAE Technical Paper, (2004)

Galindo, J., A. Tiseira, P. Fajardo, y Navarro R., Coupling methodology of 1D finite difference and 3D finite volume CFD codes based on the Method of Characteristics, doi:10.1016/j.mcm.2010.11.078, Mathematical and Computer Modelling, 54(11), 1738-1746 (2010)

Gocmez, T., y U. Deuster, An integral engineering solution for design of exhaust manifolds (No. 2009-01-1229). SAE Technical Paper, 1-12 (2009)

Gopaal, F., K. Varma, y L. Suresh, Exhaust Manifold Design-FEA Approach, International Journal of Engineering Trends an Technology (IJETT), 485-489 (2014)

Hassan, J. M., W. S. Mohammed, T. A. Mohamed, y W. H. Alawee, CFD Simulation for Manifold with Taperedlongitudinal Section. International Journal of Emerging Technology and Advanced Engineering, 4(2), 28-35 (2014)

Jeong, W., y J. Seong, Comparison of effects on technical variances of computational fluid dynamics (CFD) software based on finite element and finite volume methods, doi:10.1016/j.jimecsci.2013.10.017, International Journal of Mechanical Sciences, 78, 19-26 (2014)

Lakshmikantha, M. y M. Kec, Optimization of Exhaust System. doi:10.4271/2002-01-0059, SAE Technical Paper, (2002)

Latheesh, V. M., P. Parthasarathy, V. Baskaran, y S. Karthikeyan, Design and CFD analysis of intake port and exhaust port for a 4 valve cylinder head engine. In IOP Conference Series: Materials Science and Engineering (Vol. 310, No. 1, p. 012122). IOP Publishing (2018)

Lederer, G., E. Charkaluk, L. Verger, y A. Constantinescu, Numerical lifetime assessment of engine parts submitted to thermomechanical fatigue, application to exhaust manifold design (No. 2000-01-0789). SAE Technical Paper, (2000)

Mamiya, N., T. Masuda, y Y. Noda, Thermal fatigue life of exhaust manifolds predicted by simulation (No. 2002-01-0854). SAE Technical Paper. (2002)

Navadagi, V., y S. Sangamad, S., CFD Analysis of Exhaust Manifold of Multi-Cylinder Petrol Engine for Optimal Geometry to Reduce Back Pressure. International Journal of Engineering Research \& Technology (IJERT), 92-97 (2014)

Niyaz, H. M. y A. S. Dhekane, Twin helical intake port design optimization and validation by using cfd analysis International Journal of Emerging Technology and Advanced Engineering. 4 454-462 (2014)

Park, K. H., B. L. Choi, K. W. Lee, K. S. Kim, y Y. Y. Earmme, Modelling and design of an exhaust manifold under thermomechanical loading. doi: 10.1243/09544070D06404, Proceedings of the Institution of Mechanical Engineers, Part D: Journal of Automobile Engineering, 220(12), 1755-1764 (2006)

Patil, A., L.G. Navale y V.S. Patil, Experimental Verification and CFD Analysis of Single Cylinder Four Strokes C.I. Engine Exhaust System, ISSN: 2277-7261, Inter. J. of Science, Spirituality, Business and Tech., 3(1), 33-38 (2014)

Rocha-Hoyos, J., E. Llanes-Cedeño, S. Celi-Ortega y D. Peralta-Zurita, Efecto de la Mezcla de Biodiésel en el Rendimiento y la Opacidad del Motor Diésel. Inf. Tecnol., 30(3), en prensa (2019)

Rocha-Hoyos, J., L. Tipanluisa, V. Zambrano, y A. Portilla, Estudio de un Motor a Gasolina en Condiciones de Altura con Mezclas de Aditivo Orgánico en el Combustible. doi: 10.4067/S0718-07642018000500325, Inf. Tecnol., 29(5), 325-334 (2018) 
Rocha-Hoyos, J., Tipanluisa, L. E., Reina, S. W. y Ayabaca, C. R. Evaluación del Sistema de Tracción en un Vehículo Eléctrico Biplaza de Estructura Tubular, doi: 10.4067/S0718-07642017000200004, Inf. Tecnol. , 28(2), 29-36 (2017)

Sabareesh, N., P. Shiva, M. Achuy, A. Srinivas, DESIGN AND ANALYSIS OF EXHAUST MANIFOLD. International Journal of Advance Research in Science and Engineering, 1-7 (2017)

Santacreu, P. O., L. Bucher, A. Koster, y L. Rémy, Thermomechanical fatigue of stainless steels for automotive exhaust systems, doi:10.1051/metal:2006102 Revue de Métallurgie-International Journal of Metallurgy, 103(1), 37-42 (2006)

Selvanathan, P. S., R. Sudhakaran, K. Venkatesh, C. S. Tites, y D. Rajamanikandan, CFD analysis of IC engine exhaust manifold with respect to the performance of a turbocharger. Adv. Natural and App. Sciences, 11(4), $242-250$ (2017)

Seshadri, S., Design and CFD Analysis of the Intake Manifold for the Honda CBR250RR Engine. Master Thesis, The University of Texas at Arlington, Arlington (2015)

Srinivasa, V. K., S. Renjith, y B. Shome, Design of experiments enabled CFD approach for optimizing cooling fan performance (No. 2014-01-0658). SAE Technical Paper, (2014)

Sweta J., y A. Agrawal, Coupled Thermal - Structural Finite Element Analysis for Exhaust Manifold of an Off-road Vehicle Diesel Engine, International Journal of Soft Computing and Engineering (IJSCE), 3(4), 226-230 (2013)

Umesh, K. S., y V. P. K. Rajagopal, Cfd Analysis Of Exhaust Manifold Of Multi-Cylinder Si Engine Todetermine Optimal Geometry For Reducing Emissions. International Journal of Automobile Engineering Research and Development, 45-56 (2013)

Vaidya, V., y P. P. Hujare, Optimization of Sound Pressure Level of Air Intake System by using GT-Power, International Journal of Emerging Science and Engineering (IJESE), 2(3), 9-11 (2014)

Valderrama, J. O., Principales Aspectos sobre la Preparación de un Artículo para ser Publicado en una Revista Internacional de Corriente Principal, doi: 10.4067/S0718-07642005000200002, Inf. Tecnol., 16(2), 3-14 (2005)

Van Basshuysen, R., y F. Schäfer, Internal combustion engine handbook-basics, components, systems and perspectives, Editorial SAE, (2018)

Vega, W., E.A. Llanes-Cedeño, J. Molina, y J.C. Rocha-Hoyos, Revisión de las Características de Modelado y Optimización para el Diseño del Sistema de Suspensión Macpherson. doi:10.4067/S0718-07642018000600221, Inf. Tecnol., 29(6), 221-234 (2018)

Yoshizawa, K., K. Mori, y S. Kimura, Numerical analysis of the exhaust gas flow and heat transfer in a close-coupled catalytic converter system during warm-up, doi:10.4271/2001-01-0943, SAE Technical Paper, (2001)

Zeytin, H.K., C. Kubilay, , H. Aydin, A.A. Ebrinc, y B. Aydemir, Effect of Microstructure on Exhaust Manifold Cracks Produced From SiMo Ductile Iron, doi:10.1016/S1006-706X(09)60040-6, Journal of Iron and Steel Research, International, 16(3), 32-36 (2009) 